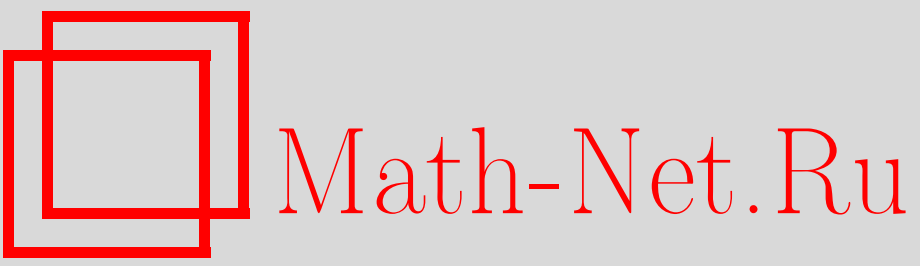

А. С. Жеданов, В. П. Спиридонов, Гипергеометрические биортогональные рациональные функции, УМН, 1999, том 54, выпуск 2, 173-174

DOI: https://doi.org/10.4213/rm150

Использование Общероссийского математического портала Math-Net.Ru подразумевает, что вы прочитали и согласны с пользовательским соглашением

http://www.mathnet.ru/rus/agreement

Параметры загрузки:

IP: 54.162 .27 .143

26 апреля 2023 г., 15:34:08 


\title{
ГИПЕРГЕОМЕТРИЧЕСКИЕ БИОРТОГОНАЛЬНЫЕ РАЦИОНАЛЬНЫЕ ФУНКЦИИ
}

\author{
А. С. ЖЕДАНОв, В.П. СПИРИ ДОНОв
}

Обозначим через $P_{n}^{j}(z), n, j \in \mathbb{Z}$, функции независимой переменной $z \in \mathbb{R}$, связанные друг с другом соотношениями

$$
\begin{aligned}
& P_{n}^{j+1}(z)=\frac{D_{n}^{j+1} P_{n+1}^{j}(z)+C_{n}^{j+1}\left(z-\alpha_{n}^{j+1}\right) P_{n}^{j}(z)}{z-\lambda_{j+1}}, \\
& P_{n}^{j-1}(z)=B_{n}^{j} P_{n}^{j}(z)+A_{n}^{j}\left(z-\beta_{n}^{j}\right) P_{n-1}^{j}(z),
\end{aligned}
$$

в которых коэффицциенты $A_{n}^{j}, \ldots, \lambda_{j}$ не зависят от $z$. Сдвинув $j \rightarrow j-1$ в (1) и исключив $P_{n}^{j-1}$ с помощью (2), получаем трехчленное рекуррентное соотношение

$$
\begin{gathered}
P_{n+1}^{j}(z)+r_{n}^{j}\left(v_{n}^{j}-z\right) P_{n}^{j}(z)+u_{n}^{j}\left(z-\alpha_{n}^{j}\right)\left(z-\beta_{n}^{j}\right) P_{n-1}^{j}(z)=0 \\
u_{n}^{j}=\frac{A_{n}^{j} C_{n}^{j}}{D_{n}^{j} B_{n+1}^{j}}, \quad r_{n}^{j}=\frac{1-D_{n}^{j} A_{n+1}^{j}-C_{n}^{j} B_{n}^{j}}{D_{n}^{j} B_{n+1}^{j}} \\
r_{n}^{j} v_{n}^{j}=\frac{\lambda_{j}-\beta_{n+1}^{j} D_{n}^{j} A_{n+1}^{j}-\alpha_{n}^{j} C_{n}^{j} B_{n}^{j}}{D_{n}^{j} B_{n+1}^{j}}
\end{gathered}
$$

Сдвинув $j \rightarrow j+1$ в (2) и исключив $P_{n}^{j+1}$ с помощью (1) приходим к (3) с другими рекуррентными коэффициентами. В качестве условий совместимости выступают ограничения $\beta_{n}^{j}=\beta_{n}, \alpha_{n}^{j}=\alpha_{n+j}$ и следуюшие нелинейные разностные уравнения:

(5) $\frac{A_{n}^{j} C_{n}^{j}}{B_{n+1}^{j} D_{n}^{j}}=\frac{A_{n}^{j+1} C_{n-1}^{j+1}}{B_{n}^{j+1} D_{n}^{j+1}}, \frac{C_{n}^{j} B_{n}^{j}+A_{n+1}^{j} D_{n}^{j}-1}{B_{n+1}^{j} D_{n}^{j}}=\frac{C_{n}^{j+1} B_{n}^{j+1}+A_{n}^{j+1} D_{n-1}^{j+1}-1}{B_{n}^{j+1} D_{n}^{j+1}}$,

(6) $\frac{\alpha_{n+j} C_{n}^{j} B_{n}^{j}+\beta_{n+1} A_{n+1}^{j} D_{n}^{j}-\lambda_{j}}{B_{n+1}^{j} D_{n}^{j}}=\frac{\alpha_{n+j+1} C_{n}^{j+1} B_{n}^{j+1}+\beta_{n} A_{n}^{j+1} D_{n-1}^{j+1}-\lambda_{j+1}}{B_{n}^{j+1} D_{n}^{j+1}}$.

Начальные условия $P_{0}^{j}(z)=p_{j}, P_{1}^{j}(z)=r_{0}^{j}\left(z-v_{0}^{j}\right)$ в (3) приводят при $n>0$ к полиномам, обобщающим ортогональные полиномы. Для самосогласованности (2) при $n=0$ наложим условие $A_{0}^{j}=A_{0}^{j} \beta_{0}=0$. Цепные дроби, ассоциированные с (3), назывались в [1] дробями ти-

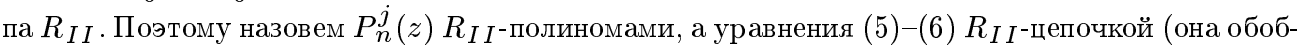
щает цепочку Тоды с дискретным временем, cp. [2]). Как показано в $[1], R_{I I}$-полиномы служат основой для построения биортогональных рациональных функций, так как для каждого $j$ существует линейный функционал $\mathscr{L}_{j}$ такой, что $\mathscr{L}_{j}\left[z^{m} P_{n}^{j}(z) / \prod_{k=1}^{n}\left(z-\alpha_{j+k}\right)\left(z-\beta_{k}\right)\right]=0$ для $0 \leqslant m<n$. В [3] это соотношение представлено в виде условия биортогональности двух рациональых функций. Обозначим $R_{0}^{j}(z)=S_{0}^{j}(z)=1, R_{n}^{j}(z)=P_{n}^{j}(z) / \prod_{k=1}^{n}\left(z-\alpha_{j+k}\right)$, $S_{n}^{j}(z)=P_{n}^{j}(z) / \prod_{k=1}^{n} u_{k}^{j}\left(z-\beta_{k}\right)$. Определим также другую последовательность рациональных функций $H_{0}^{j}(z)=u_{1}^{j} S_{1}^{j}(z)-r_{0}^{j}$ и $H_{n}^{j}(z)=u_{n+1}^{j} S_{n+1}^{j}(z)-r_{n}^{j} S_{n}^{j}(z)+S_{n-1}^{j}(z)$ для $n>0$.

ТЕОрема 1. Функиия $R_{n}^{j}(z)$ ортогональна $H_{m}^{j}(z): \mathscr{L}_{j}\left[H_{m}^{j} R_{n}^{j}\right]=0$ при $n \neq m$.

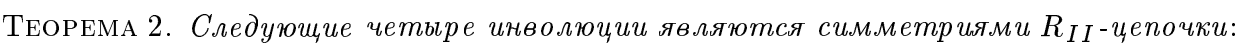

Работа выполнена при финансовой поддержке Российского фонда фундаменталњных исследований (грант № 97-01-00281) и ИНТАС (грант № 96-0700). 
1. $\tilde{A}_{n}^{j}=D_{-n}^{-j}, \tilde{D}_{n}^{j}=A_{-n}^{-j}, \tilde{B}_{n}^{j}=C_{-n}^{-j}, \tilde{C}_{n}^{j}=B_{-n}^{-j}, \tilde{\beta}_{n}=\beta_{1-n}, \tilde{\alpha}_{n+j}=\alpha_{-n-j}$, $\tilde{\lambda}_{j}=\lambda_{-j}$

2. $\tilde{A}_{n}^{j}=\frac{1}{A_{j}^{n}}, \tilde{B}_{n}^{j}=\frac{B_{j}^{n}}{A_{j}^{n}}, \tilde{C}_{n}^{j}=\frac{C_{j-1}^{n+1}}{D_{j-1}^{n+1}}, \tilde{D}_{n}^{j}=\frac{1}{D_{j-1}^{n+1}}, \tilde{\alpha}_{n+j}=\alpha_{n+j}, \tilde{\beta}_{n}=\lambda_{n}, \tilde{\lambda}_{j}=\beta_{j}$;

3. $\tilde{A}_{n}^{j}=\frac{A_{n}^{1-j-n}}{B_{n}^{1-j-n}}, \tilde{B}_{n}^{j}=\frac{1}{B_{n}^{1-j-n}}, \tilde{C}_{n}^{j}=\frac{1}{C_{n}^{1-j-n}}, \tilde{D}_{n}^{j}=\frac{D_{n}^{1-j-n}}{C_{n}^{1-j-n}}, \tilde{\lambda}_{j}=\alpha_{1-j}, \tilde{\alpha}_{j+n}=$ $\lambda_{1-j-n}, \tilde{\beta}_{n}=\beta_{n}$

4. $\tilde{A}_{n}^{j}=B_{1-n-j}^{j}, \tilde{B}_{n}^{j}=A_{1-n-j}^{j}, \tilde{C}_{n}^{j}=D_{-n-j}^{j}, \tilde{D}_{n}^{j}=C_{-n-j}^{j}, \tilde{\alpha}_{n+j}=\beta_{1-n-j}$, $\tilde{\beta}_{n}=\alpha_{1-n}, \tilde{\lambda}_{j}=\lambda_{j}$.

Эти инволюции могут качественно изменять форму решений $R_{I I}$-цепочки, но существует такой подкласс, для которого этого не происходит. Анализ возможной структуры таких решений привел к следующему анзацу обобщенного разделения переменных (ср. [2])

$$
\begin{gathered}
A_{n}^{j}=\frac{d(n) \rho(2 j+n)}{g(2 n+j) g(2 n+j-1) \phi(n-j) \phi(n-j-1)}, \quad B_{n}^{j}=1, \\
C_{n}^{j}=\frac{c(n+j) \phi(n-j) \phi(n-j+1)}{\sigma(j) g(2 n+j) g(2 n+j+1)}, \quad D_{n}^{j}=\frac{\rho(2 j+n) \phi(n-j) \phi(n-j+1)}{\sigma(j)},
\end{gathered}
$$

где $d(0)=0$. Перенормировка $P_{n}^{j}(z) \rightarrow \xi_{n}^{j} P_{n}^{j}(z)$ позволяет придать уравнениям (1)-(6) различные формы. Воспользовавшись этой свободой, мы выбрали калибровку $B_{n}^{j}=1, P_{0}^{j}=1$. Нетрудно проверить, что (7) автоматически удовлетворяет первому уравнению в (5) для произвольных функций $d(x), \ldots, \sigma(x)$. Инволюции приводят к перестановкам функций $d(x), c(x), \sigma(x)$ между собой с точностью до линейных преобразований аргументов. Аналогичная ситуация имеет место для $\rho(x), \phi(x), g(x)$. Поэтому можно предположить, что соответствующие функции имеют одинаковую форму. Эти наблюдения позволили найти следующее явное решение $R_{I I}$-цепочки. Опуская несушественные общие нормировочные множители, имеем: $g(x)=x-g_{0}, \rho(x)=$ $x-g_{0}+\phi_{0}, \phi(x)=x-\phi_{0}, c(x)=d\left(g_{0}-x\right), \sigma(x)=d\left(x+\phi_{0}\right)$, где $g_{0}, \phi_{0}-$ произвольные постоянные и $d(x)$ - полином 6 -й степени с любопытным соотношением между его корнями: $d(x)=x \prod_{k=1}^{5}\left(x-d_{k}\right), \sum_{k=1}^{5} d_{k}=1+2\left(\phi_{0}+g_{0}\right)$, оставляюшим 4 свободных параметра. При этом $\lambda_{j}=e\left(j+\phi_{0}\right) / d\left(j+\phi_{0}\right), \beta_{n}=e(n) / d(n), \alpha_{n}=e\left(g_{0}-n\right) / d\left(g_{0}-n\right)$, где $e(x)$ - полином, аналогичный $d(x): e(x)=x \prod_{k=1}^{5}\left(x-e_{k}\right), \sum_{k=1}^{5} e_{k}=1+2\left(g_{0}+\phi_{0}\right)$. Для удобства сопоставления с результатами [4] обозначим $s \equiv j+2-g_{0}, a \equiv 2 j+1+\phi_{0}-g_{0}$.

Теорема 3. Рекуррентное соотношение (3) для найденного рачионального решения

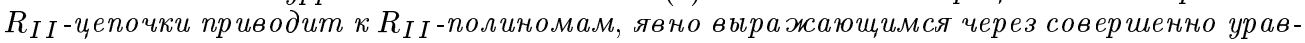
новешенный, 2-сбалансированный гипергеометрический ряд ${ }_{9} F_{8}$ :

$$
P_{n}^{j}(z)=f_{n}^{j}(z)_{9} F_{8}\left(\begin{array}{c}
a, a / 2+1,-n, s+n-1, a+2-s-x_{1}, \ldots, a+2-s-x_{5} \\
a / 2, a+n+1, a+2-s-n, s-1+x_{1}, \ldots, s-1+x_{5}
\end{array} ;\right),
$$

$$
f_{n}^{j}(z)=\frac{(1-z)^{n} \prod_{k=1}^{5}\left(s-1+x_{k}\right)_{n}}{(n+s-1)_{n}(a+1)_{n}},
$$

где $x_{1}(z), \ldots, x_{5}(z)$ - корни уравнения 5 -й степени $z \prod_{k=1}^{5}\left(x-d_{k}\right)=\prod_{k=1}^{5}\left(x-e_{k}\right)$.

Функции, найденные Вильсоном в [4], соответствуют случаю, когда $\beta_{n}, \alpha_{n}, \lambda_{n}$ являются полиномами 2-й степени, возникающими при предельном переходе в $d(n)$ к полиному 4 -й степени такому, что его корни совпадают с 4 корнями $e(n)$.

\section{СПИСОК ЛИТЕРАТУРЫ}

[1] Ismail M. E. H., Masson D. R. // J. Approx. Theory. 1995. V. 83. P. 1-40. [2] Spiridonov V., Zhedanov A. // J. Phys. A: Math. Gen. 1997. V. 30. P. 8727-8737. [3] Zhedanov A. // Preprint CRM-2539 1998. [4] Wilson J. A. // SIAM J. Math. Anal. 1991. V. 22 № 4. P. $1147-1155$. 